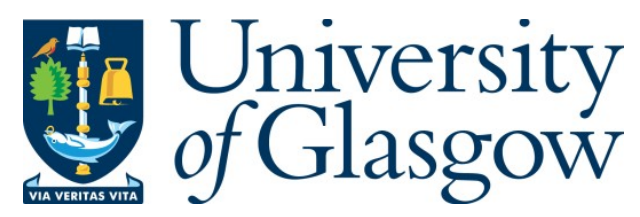

Fernando, S. M., Deane, F. P., McLeod, H. J., and Davis, E. L. (2017) Carer burden and stigma in schizophrenia and affective disorders: experiences from Sri Lanka. Asian Journal of Psychiatry, 26, pp. 77-81.

There may be differences between this version and the published version. You are advised to consult the publisher's version if you wish to cite from it.

http://eprints.gla.ac.uk/134868/

Deposited on: 19 January 2017

Enlighten - Research publications by members of the University of Glasgow http://eprints.gla.ac.uk 
A Carer burden and stigma in Schizophrenia and Affective Disorders: Experiences

\section{from Sri Lanka}

Sunera M. Fernando ${ }^{1}$, Frank P. Deane ${ }^{2 *}$, Hamish J. McLeod ${ }^{3}$, Esther L. Davis ${ }^{2}$

${ }^{1}$ Department of Psychological Medicine, University of Colombo, Sri Lanka

${ }^{2}$ Illawarra Institute of Mental Health, School of Psychology, University of Wollongong,

Australia

${ }^{3}$ Institute of Health and Wellbeing, College of Medical, Veterinary, and Life Sciences, University of Glasgow, United Kingdom

*Corresponding author:

Address: Illawarra Institute of Mental Health (B22), Northfields Avenue, University of

Wollongong, Wollongong NSW 2522

Phone: +61242214523

Fax: +61242215585

Email: fdeane@uow.edu.au 


\begin{abstract}
Objectives: Stigma compounds the burden experienced by family members of those with a mental illness. This study aimed to examine burden experienced by carers of people with schizophrenia or affective disorders and to explore the relationship between carer burden and stigma.

Method: A cross sectional descriptive study was conducted with patient-carer dyads involving 67 patients diagnosed with schizophrenia and 51 diagnosed with affective disorder. Carers completed the Zarit Burden Interview (short version) and stigma was measured using the Stigma Scale and the Internalised Stigma of Mental Illness Scale.

Results: Carer burden was significantly higher for schizophrenia than affective disorders. Female carers experienced significantly higher burden than male carers. Diagnosis, gender of carer and stigma predicted $21 \%$ of the variance in carer burden, with gender and carer disclosure of mental illness identified as significant predictors.

Conclusions: Reducing stigma related to disclosure of mental illness in carers has the potential to reduce carer burden.
\end{abstract}

Keywords: stigma, carer burden, schizophrenia, affective disorders, Sri Lanka 


\section{Introduction}

Carer burden refers to the, "total experience of caring for the ill relative including effects on physical, psychological and social well being as well as the capacity to cope and adjust to that circumstance" (Ohaeri, 2003, p. 458). Family members play an integral role as informal carers for the person with mental illness and have been consistently reported to experience high levels of carer burden (Caqueo-Urizar \& Gutierrez-Maldonado, 2006; Tsang, Tam, Chan, \& Cheung, 2003).

In Sri Lanka, the family plays an important role in looking after a patient with mental illness. This role is amplified by the cultural expectation that families look after their children well into adulthood and a scarcity of social support institutions. However, the extent to which looking after a relative with mental illness leads to carer burden in Sri Lanka has only been documented in two studies to date. An early study of 50 Sri Lankan carers of patients with psychotic illnesses reported that $60 \%$ of carers felt anxious and depressed and $54 \%$ felt their workload increased due to caring for their relative with mental illness (de Silva \& de Silva, 2001). However, this study did not examine factors impacting on carer burden, which are important in order to identify potential strategies for providing effective support. A more recent study conducted among 80 Sri Lankan carers of patients with schizophrenia and bipolar affective disorder found that higher strain was associated with spending more time with the patient, assault by the patient, family disputes, and disruption to employment (Rodrigo, Fernando, Rajapakse, De Silva, \& Hanwella, 2013).

No studies to date have examined potential differential carer burden associated with different mental health diagnoses in Sri Lanka. There are conflicting results regarding the differential burden associated with a caring for patients with schizophrenia and depression in developed countries. For example, a study conducted in five European countries involved 260 carers of patients with depression and 342 carers of patients with schizophrenia and 
found carers of patients with schizophrenia reported significantly higher burden than carers of patients with depression (van Wijngaarden et al., 2004). Contrasting this finding, a subsequent study conducted in three European countries revealed no significant difference in carer burden between 252 carers of patients with depression and 151 carers of patients with schizophrenia (van Wijngaarden et al., 2009). Thus, although previous research in this area has provided a useful start in understanding the extent of and some correlates of carer burden, there is a need for additional research that further clarifies potential contributors to carers' burden in Sri Lanka.

Stigma is a notable factor implicated in carer burden that has not been examined for carers in Sri Lanka. While stigma clearly adversely affects the quality of life of people with mental illness (Link, Cullen, Frank, \& Wozniak, 1987; Mathias, Kermode, San Sebastian, Koschorke, \& Goicolea, 2015), it also substantially impacts family members and friends (Corrigan, 2005; Yin et al., 2014). Goffman (1963) called this phenomenon "courtesy stigma"; defined as a situation in which both the stigmatised person and their family members are treated as one unit in social situations. Stigma has been known to contribute to carer burden by restricting the family's access to sources of help (Tsang et al., 2003). Results from a study involving 427 Chinese carers revealed that nearly three-quarters of carers felt they did not have the support of friends and the experience of stigma was negatively associated with perceived social support (Yin et al., 2014). Notably, a recent survey conducted among 119 Sri Lankan carers of patients with depression or schizophrenia found that carers hold stigmatising attitudes towards people with mental illness (Ediriweera, Fernando, \& Pai, 2012). For example, the majority agreed that the mental illness was a sign of personal weakness of the person, while half perceived the person with the mental illness as erratic, and just less than half that they were dangerous (Ediriweera et al., 2012). A greater understanding about 
the stigma experiences of Sri Lankan carers would provide critical information about unique problems in Sri Lanka for which targeted support services can be offered.

The primary aim of this study was to examine stigma experienced by patients with schizophrenia or affective disorder and their carers, and to determine the relationship of stigma to carer burden in Sri Lanka. Recruiting patient-carer dyads allows the opportunity to assess two perspectives of stigma from persons within the same family. It is also of value to examine the impact of stigma on carer burden in the Sri Lankan context since findings from studies conducted in developed nations cannot be directly applied to developing countries such as Sri Lanka which has a highly different socio-economic environment, support systems and levels of medical care (Ediriweera et al., 2012).

\section{Method}

\subsection{Participants}

A convenience sample of outpatients was recruited from two psychiatric hospitals in Sri Lanka. The inclusion criteria were that patients were: diagnosed with schizophrenia, depression or bipolar affective disorder as defined by ICD-10 criteria (World Health Organisation, 1992); not experiencing an acute relapse of their mental illness or current episode of depression as defined by ICD-10 criteria, and; did not have a cognitive deficit or other condition identified by the treating psychiatrist that could interfere with their capacity to give informed consent. The diagnosis was ascertained from patient records made by the consultant psychiatrist.

In addition, all participants had to have been diagnosed with the mental illness for a minimum of one year and were accompanied to the hospital by their family carer. A carer was defined as the relative who spends the greatest number of hours in contact with the patient at home. Non-family carers (e.g. live-in hired help) were not eligible for this study. 
The study was reviewed and approved by the Human Research Ethics Committees of the University of Wollongong, the Faculty of Medicine of the University of Colombo, and the National Hospital of Sri Lanka. Participation was voluntary.

\subsection{Procedure}

Data was collected in separate interviews with patients and their carers when they attended a psychiatric out-patient clinic appointment. All interviews were conducted between April 2008 to September 2008 and were completed by the Principal Investigator and a trained Research Assistant.

\subsection{Measures}

Reliable and valid measures used in previous international stigma and carer burden research were translated into the Sinhalese by a bilingual translator and back into English by another translator to check for loss of meaning in the interpretation process. Following this procedure, only minor grammatical changes were required to eliminate ambiguities identified during the translation process.

\subsubsection{Measures of Carer Burden}

Carer burden was measured using the 12-item short version of the Zarit Burden Interview (Bédard et al., 2001), which has been used effectively in previous cross-cultural research (e.g., Prince et al., 2004). Responses are provided on a Likert scale ranging from 0 (never) to 4 (nearly always). The Zarit Burden Interview has been found to have good internal reliability ( $\alpha=0.88$; Bédard et al., 2001) and Cronbach's alpha was satisfactory in the current study ( $\alpha=0.78$ ). A cut off point of 17 or more is used to indicate high burden (Bédard et al., 2001). 


\subsubsection{Measures of Stigma}

Patient's perception of public stigma was measured using the Discrimination and Disclosure subscales from King et al.'s (2007) 28-item self-report Stigma Scale. Each item is rated on a 5-point Likert scale ranging from 0 (strongly disagree) to 4 (strongly agree). The items comprising the Discrimination subscale assess discrimination in education, by employers, police and health care workers (e.g. "I have been discriminated against by employers because of my mental health problem"). The Disclosure subscale assesses worry about disclosure, feeling embarrassed about disclosure, and concealment of mental health problems (e.g. "I worry about telling people that I take medicines for mental health problems"). Given that mental illness stigma significantly affects marriage Asian countries (Thara \& Srinivasan, 2000) an item on disclosure and marriage was added ("I feel the stigma of mental illness has spoiled my chances of getting married"). Internal consistency of the patient Discrimination subscale was low $(\alpha=.57)$, while internal consistency of the Disclosure subscale was satisfactory $(\alpha=.76)$.

The carers' perceptions of public stigma were assessed using a modified version of the Stigma Scale (King et al., 2007). The items were exactly the same except for rewording to make the item about the carer's own personal experience of stigma as a consequence of their relative's mental illness (e.g., "I have been discriminated against in education because of their mental health problem"). The Disclosure subscale of the Stigma Scale assessed the carer's feelings about disclosure of their relative's mental illness. These included items that ask about feeling embarrassed about disclosure, and hiding their relative's mental health problems (e.g., "I feel the need to hide my relative's mental health problems from our friends"). An equivalent item on disclosure and marriage was added as for the patient questionnaire. Internal consistency of the carer Discrimination subscale was adequate $(\alpha=.69)$, while internal consistency of the Disclosure subscale was satisfactory $(\alpha=.75)$. 
Patient self stigma was measured using 10 items selected from the Internalised Stigma of Mental Illness Scale (Ritsher, Otilingam, \& Grajales, 2003). Items are rated on a 4-point Likert scale from 1 (strongly disagree) to 4 (strongly agree). The full 29 item scale has been shown to have high internal consistency $(\alpha=.90)$ and test-retest reliability (e.g., $r=.92)$

(Ritsher et al., 2003). The high internal consistency suggested item redundancy, and thus with the need for brevity in the current study, 10 items were selected from the original scale based on the following criteria: factor loading of more than 0.5 , highest loading across factors (i.e., must not load higher on another factor), and items grouped in the factor "a priori" by Ritsher et al (2003). This criterion was used also as an indicator of face validity. Internal consistency of the 10 -item version in the current study was satisfactory $(\alpha=.73)$.

\subsection{Analyses}

Results were analysed using SPSS Statistics for Windows (SPSS Inc., 2008).

Data were examined for outliers and casewise diagnostics were used to detect any problematic cases, of which none were found. The Durbin-Watson statistic did not indicate the presence of autocorrelation and collinearity diagnostic tests revealed no issues. Normality plots and statistical tests of normality were inspected. Results showed that most burden and stigma variables had statistically significant skewness (range .45 to 1.01) and/or kurtosis (range .90 to 2.31). Transformations did not satisfactorily improve the distributions and also make interpretation more difficult, thus nonparametric analyses and bootstrapping were used where appropriate. The following analyses were conducted. First, descriptives of carer burden for different diagnoses were provided.. Second, two-tailed Spearman's correlations amongst diagnosis, gender of carer, carer burden and stigma variables (patient self stigma, patient discrimination, patient disclosure, carer discrimination, and carer disclosure subscales) were conducted. Third, differences detected between SZ and AD carers were 
examined using Chi-Square analysis and interactions between the differences and diagnosis were explored using ANOVA with bootstrapping (5,000 samples). Fourth, a regression analysis with bootstrapping (5,000 samples) was used to test predictors of carer burden. All variables that were significantly correlated with carer burden were entered in one block.

For all analyses, a p-value of $<0.05$ was considered statistically significant.

\section{Results}

\subsection{Participants}

The detailed demographics of the carer and patient samples are provided in Table 1. The study population was made up of 67 (56.78\%) patient-carer dyads where schizophrenia was the diagnosis (Sz) and 51 (43.22\%) dyads where affective disorders (AD) were diagnosed. Of the Sz carers 43 (64.18\%) were female and 31 (60.78\%) of AD carers were female. The mean duration of illness in Sz patients was 15.00 years and 11.80 years in AD patients. The mean number of hospital admissions in the last three years was 2.27 for $\mathrm{Sz}$ patients and 1.90 for AD patients. Analyses revealed no significant differences between diagnostic groups on the demographic variables.

[INSERT TABLE 1 HERE]

\subsection{Carer burden}

Results indicated that $29(43 \%)$ of Sz carers and $10(20 \%)$ of AD carers met criterion for high carer burden. This difference was statistically significant, indicating a greater proportion of Sz carers had high burden compared to $\mathrm{AD}$ carers $\left(\chi_{1,118}^{2}=13.56, \mathrm{p}<.001\right.$. There was also a gender difference with greater burden in female carers (41.9\%) compared to male carers $\left(18.2 \% ; \chi^{2}{ }_{1,118}=7.62, \mathrm{p}<.05\right)$. 
To determine whether there was an interaction between diagnosis (schizophrenia/affective disorder) and carer gender (male/female) a 2 x 2 ANOVA with bootstrapping (5,000 samples) was conducted on burden. There was no significant interaction between diagnosis and gender.

There was a statistically significant main effect for both gender and diagnosis. When the main effect for diagnosis was considered, Sz carers reported higher levels of carer burden $(M=1.38, S D=.65)$ than $\mathrm{AD}$ carers $\left(M=1.11, S D=0.47 ; F_{1,114}=4.19, M S E=.09, p=.02\right.$, $95 \%$ CI $[.04, .40])$. The main effect for gender showed that female carers reported higher levels of carer burden $(M=1.38, S D=.66)$ than male carers $\left(M=1.06, S D=.40 ; F_{1,114}=7.61\right.$, $M S E=.09, p<.01,95 \%$ CI $[.12, .48])$.

Multiple regression analysis was conducted in order to identify predictors of carer burden. Correlation analyses indicated that carer burden was significantly correlated with the patient disclosure and carer disclosure subscales, which were consequently included in the regression (see Table 2).

\section{[INSERT TABLE 2 HERE]}

Results from the multiple regression analysis indicated that diagnosis, gender of carer, and stigma variables (patient disclosure and carer disclosure subscales) accounted for a significant $22 \%$ of the variability in carer burden (adjusted $R^{2}=0.22, F_{4,113}=9.31 p<0.001$ ). Gender was identified as a unique predictor (see Table 3).

\section{Discussion}

The results suggest that there may be unique profiles of carer burden dependent on the type of disorder and common challenges associated with caring for someone with those 
disorders. This is important information for identifying and supporting carers who are at risk of high carer burden in Sri Lanka. It was found that female carers experienced significantly higher levels of burden than male carers, which is in keeping with international studies (e.g., Friedrich et al., 2015; Moeller-Leimkuehler \& Wiesheu, 2012; Ricard, Bonin, \& Ezer, 1999). A review found that increased psychiatric morbidity amongst female carers may be attributed to differing levels in care involvement, with female carers found to generally spend more time providing care and more likely to assist with day-to-day household-related and personal care tasks (Yee \& Schulz, 2000). Gender role expectations that females should assume the role of primary carer, which can conflict with other life ambitions such as career, has also been suggested as an additional source of burden for female carers (Montgomery, 1992; Yee \& Schulz, 2000).

The finding that carer burden scores were significantly higher for Sz carers than AD carers is consistent with previous research conducted in Europe (van Wijngaarden et al., 2004). Similarly, a study conducted in the US found that Sz carers showed higher levels of economic burden and lower levels of quality of life compared to carers of other conditions (e.g., bipolar disorder, dementia, epilepsy; Csoboth, Witt, Villa, \& O'Gorman, 2015). A comparison between Sz carers and those of patients with Bipolar Disorder in India found that Sz carers were more likely to report being less supported by family and friends, experiencing more relationship difficulties with family and friends, and having a disrupted routine (Vasudeva, Sekhar, \& Rao, 2013). One way to clarify the source of burden differences for different diagnostic groups might be to inspect items within the Zarit Burden Interview. For example, Sz carers admitted to getting more angry with their relative (approximately 36\%) than AD carers (approximately 20\%). This may reflect higher levels of expressed emotion in Sz carers - a factor that has been linked to increased risk of relapse amongst patients with schizophrenia (Hooley, 2007; Marom, Munitz, Jones, Weizman, \& Hermesh, 2005) and 
higher levels of carer burden (Caqueo-Urizar et al., 2014; Magliano et al., 1998; Scazufca \& Kuipers, 1996). Thus these results suggest a potential need to provide different types and levels of support to carers depending on the unique needs arising from different diagnoses.

The identification of stigma as a correlate of burden provides some evidence for considering interventions that reduce stigma. These might include strategies to increase empowerment and autonomy of carers may be useful (Lefley, 2009). For example, advocacy groups are an effective means to change major socio-structural and attitudinal barriers to achieve better mental health and support for patients and their families (World Health Organisation, 2003). The WHO realises the importance of developing advocacy movements in countries and asserts it as a major intervention to improve mental health care (Funk, Minoletti, Drew, Taylor, \& Saraceno, 2006). Such developments are likely to initially need government and public sector support in Sri Lanka. Consumer support groups may also be useful, as participation has been shown to be effective to reduce carer burden in families (Chou, Pu, Lee, Lin, \& Kröger, 2009; Winefield \& Harvey, 1995). Although the regression results indicated that carer stigma concerns related to disclosure of mental illness contributed to the overall prediction of burden, it only approached significance $(p=.055)$ in terms of unique variance. Instead it seems that being a female carer is one of the primary predictors of perceived burden.

Notably, the mean Zarit burden interview scores in the present study were higher than a US study of AD carers (M=1.0; Martire et al., 2009), but lower than Sz carers in Japan $(\mathrm{M}=2.02$; Fujino \& Okamura, 2009; $\mathrm{M}=1.56$; Hanzawa, Tanaka, Inadomi, Urata, \& Ohta, 2008). Ratings from studies in nations of similar economic wealth such as Chile were also higher (M=3.86; Caqueo-Urizar \& Gutierrez-Maldonado, 2006). In summary, overall carer burden amongst carers supporting people with severe mental illness tended to be lower in Sri Lanka than in other countries using the same standardised measure. It is possible that this 
occurred because there is greater family support (extended family structures) available in Sri Lanka. It may also reflect relatives' beliefs that the care role is an integral and valued role and thus is not viewed as burdensome compared to samples from other countries. However, these are speculations and further research is needed to elucidate the exact reasons for this reported level of carer burden in Sri Lanka.

A key limitation of this study is the cross sectional design from which is cannot be distinguished whether experiences of stigma cause increased burden or whether those who feel more burden interpret experiences as being more stigmatising. Longitudinal studies are needed to assess the long-term outcomes of stigma on caregiving and the changes that occur with time. In addition, data is unavailable regarding those patients and carers who refused participation and thus the sample may not be representative of all patients and carers in Sri Lanka. Nonetheless, this study has provided an initial examination of burden profiles of $\mathrm{Sz}$ and AD carers..

\subsection{Conclusions}

Levels of carer burden were found to be significantly higher for Sz than AD carers and in female than male carers. Gender and to some extent stigma related to disclosure of mental illness by carers were identified as predictors of burden. In international comparisons, carer burden tended to be lower in Sri Lanka than in other countries. The results provide important information from which to further explore unique sources of burden for carers in Sri Lanka and ultimately the provision of targeted support. 


\section{Acknowledgements}

The study team is grateful to the Sri Lankan patients and their carers who gave their time to participate in this study. 


\section{References}

Bédard, M., Molloy, D. W., Squire, L., Dubois, S., Lever, J. A., \& O'Donnell, M. (2001). The Zarit Burden Interview: A new short version and screening version. Gerontologist, 41(5), 652-657. doi: 10.1093/geront/41.5.652

Caqueo-Urizar, A., \& Gutierrez-Maldonado, J. (2006). Burden of care in families of patients with schizophrenia. Qual Life Res, 15(4), 719-724. doi: 10.1007/s11136-005-4629-2

Caqueo-Urizar, A., Miranda-Castillo, C., Lemos Giraldez, S., Maturana, S.-1. L., Ramirez Perez, M., \& Mascayano Tapia, F. (2014). An updated review on burden on caregivers of schizophrenia patients. Psicothema, 26(2), 235-243. doi:

10.7334/psicothema2013.86

Chou, Y. C., Pu, C. Y., Lee, Y. C., Lin, L. C., \& Kröger, T. (2009). Effect of perceived stigmatisation on the quality of life among ageing female family carers: a comparison of carers of adults with intellectual disability and carers of adults with mental illness. J Intell Disabil Res, 53(7), 654-664. doi: 10.1111/j.1365-2788.2009.01173.x

Corrigan, P. W. (2005). On the Stigma of Mental Illness: Practical Strategies for Research and Social Change. Washington, DC: American Psychological Association.

Csoboth, C., Witt, E. A., Villa, K. F., \& O'Gorman, C. (2015). The humanistic and economic burden of providing care for a patient with schizophrenia. Int J Soc Psychiatr, 61(8), 754-761.

de Silva, D., \& de Silva, S. (2001). A preliminary study of the impact of long-term psychotic disorder on patients' families. Ceylon Med J, 46(4), 121-123.

Ediriweera, H. W., Fernando, S. M., \& Pai, N. B. (2012). Mental health literacy survey among Sri Lankan carers of patients with schizophrenia and depression. Asian J Psychiatry, 5(3), 246-250. doi: 10.1016/j.ajp.2012.02.016 
Friedrich, F., Gross, R., Wrobel, M., Klug, G., Unger, A., Fellinger, M., . . Wancata, J. (2015). Burden of mothers and fathers of persons with Schizophrenia. Psychiat Prax, 42(4), 208-215. doi: 10.1055/s-0034-1370080

Fujino, N., \& Okamura, H. (2009). Factors Affecting the Sense of Burden Felt by Family Members Caring for Patients With Mental Illness. Arch Psychiat Nurs, 23(2), 128-137. doi: 10.1016/j.apnu.2008.05.006

Funk, M., Minoletti, A., Drew, N., Taylor, J., \& Saraceno, B. (2006). Advocacy for mental health: roles for consumer and family organizations and governments. Health Promot Int, 21(1), 70-75. doi: 10.1093/heapro/dai031

Goffman, E. (1963). Stigma: Notes on Management of Spoiled Identity. Englewood Cliffs, NJ: Prentice-Hall.

Hanzawa, S., Tanaka, G., Inadomi, H., Urata, M., \& Ohta, Y. (2008). Burden and coping strategies in mothers of patients with schizophrenia in Japan. Psychiat Clin Neuros, 62(3), 256-263. doi: 10.1111/j.1440-1819.2008.01791.x

Hooley, J. M. (2007). Expressed emotion and relapse of psychopathology. Annu Rev Clin Psycho, 3, 329-352. doi: 10.1146/annurev.clinpsy.2.022305.095236

King, M., Dinos, S., Shaw, J., Watson, R., Stevens, S., Passetti, F., . . Serfaty, M. (2007). The Stigma Scale: Development of a standardised measure of the stigma of mental illness. Brit J Psychiatry, 190, 248-254. doi: 10.1192/bjp.bp.106.024638

Lefley, H. P. (2009). A psychoeducational support group for serious mental Iilness. $J$ Spec Group Work, 34(4), 369-381. doi: 10.1080/01933920903219094

Link, B. G., Cullen, F. T., Frank, J., \& Wozniak, J. F. (1987). The social rejection of former mental patients: Understanding why labels matter. Am J Sociol, 92(6), 1461-1500. doi: $10.1086 / 228672$ 
Magliano, L., Fadden, G., Economou, M., Xavier, M., Held, T., Guarneri, M., . . Maj, M. (1998). Social and clinical factors influencing the choice of coping strategies in relatives of patients with schizophrenia: results of the BIOMED I study. Soc Psych Psych Epi, 33(9), 413-419.

Marom, S., Munitz, H., Jones, P. B., Weizman, A., \& Hermesh, H. (2005). Expressed emotion: Relevance to rehospitalization in schizophrenia over 7 years. Schizophrenia Bull, 31(3), 751-758. doi: 10.1093/schbul/sbi016

Martire, L. M., Hinrichsen, G. A., Morse, J. Q., Reynolds, C. F., III, Gildengers, A. G., Mulsant, B. H., . . Kupfer, D. J. (2009). The Mood Disorder Burden Index: A scale for assessing the burden of caregivers to adults with unipolar or bipolar disorder. Psychiat Res, 168(1), 67-77. doi: 10.1016/j.psychres.2008.04.010

Mathias, K., Kermode, M., San Sebastian, M., Koschorke, M., \& Goicolea, I. (2015). Under the banyan tree: Exclusion and inclusion of people with mental disorders in rural North India. Bmc Public Health, 15. doi: 10.1186/s12889-015-1778-2

Moeller-Leimkuehler, A. M., \& Wiesheu, A. (2012). Caregiver burden in chronic mental illness: the role of patient and caregiver characteristics. Eur Arch Psy Clin N, 262(2), 157-166. doi: 10.1007/s00406-011-0215-5

Montgomery, R. J. V. (1992). Gender differences in patterns of child-parent caregiving relationships. In J. W. Dwyer \& R. T. Coward (Eds.), Gender, families, and elder care. Newbury Park, CA: Sage.

Ohaeri, J. U. (2003). The burden of caregiving in families with a mental illness: A review of 2002. Curr Opin Psychiatr, 16(4), 457-465. doi:

10.1097/01.yco.0000079212.36371.c0 
Prince, M., Quraishi, S., Copeland, J., Varghese, M., Murali, T., Srinivasan, L., . . Olebuezie, F. (2004). Care arrangements for people with dementia in developing countries. Int J Geriatr Psych, 19(2), 170-177. doi: 10.1002/gps.1046

Ricard, N., Bonin, J. P., \& Ezer, H. (1999). Factors associated with burden in primary caregivers of mentally ill patients. Int J Nurs Stud, 36(1), 73-83. doi: 10.1016/s00207489(98)00060-1

Ritsher, J. B., Otilingam, P. G., \& Grajales, M. (2003). Internalized stigma of mental illness: Psychometric properties of a new measure. Psychiat Res, 121(1), 31-49.

Rodrigo, C., Fernando, T., Rajapakse, S., De Silva, V., \& Hanwella, R. (2013). Caregiver strain and symptoms of depression among principal caregivers of patients with schizophrenia and bipolar affective disorder in Sri Lanka. Int J Ment Health Syst, 7. doi: $10.1186 / 1752-4458-7-2$

Scazufca, M., \& Kuipers, E. (1996). Links between expressed emotion and burden of care in relatives of patients with schizophrenia. Brit J Psychiatry, 168(5), 580-587. doi: 10.1192/bjp.168.5.580

SPSS Inc. (2008). SPSS Statistics for Windows. Chicago: SPSS Inc.

Thara, R., \& Srinivasan, T. N. (2000). How stigmatising is schizophrenia in India? Int J Soc Psychiatr, 46(2), 135-141. doi: 10.1177/002076400004600206

Tsang, H. W. H., Tam, P. K. C., Chan, F., \& Cheung, W.-M. (2003). Sources of burdens on families of individuals with mental illness. Int J Rehab Res, 26(2), 123-130. doi: 10.1097/01.mrr.0000070761.13531.a1

van Wijngaarden, B., Koeter, M., Knapp, M., Tansella, M., Thornicroft, G., VazquezBarquero, J.-L., \& Schene, A. (2009). Caring for people with depression or with schizophrenia: Are the consequences different? Psychiat Res, 169(1), 62-69. doi: 10.1016/j.psychres.2008.06.013 
Vasudeva, S., Sekhar, C. K., \& Rao, P. G. (2013). Caregivers burden of patients with Schizophrenia and Bipolar Disorder: A sectional study. Indian J Psychol Med, 35(4), 352-357. doi: $10.4103 / 0253-7176.122224$

Winefield, H. R., \& Harvey, E. J. (1995). Tertiary prevention in mental-health care: Effects of group meetings for family caregivers. Aust NZ J Psychiat, 29(1), 139-145. doi: $10.3109 / 00048679509075903$

World Health Organisation. (1992). International Classification of Mental and Behavioural Disorderes: Clinical Descriptions and Diagnostic Guidelines. Geneva: World Health Organisation.

World Health Organisation. (2003). Advocacy for mental health: Mental health policy and service guidance package. Geneva: World Health Organisation.

Yee, J. L., \& Schulz, R. (2000). Gender differences in psychiatric morbidity among family caregivers: A review and analysis. Gerontologist, 40(2), 147-164.

Yin, Y., Zhang, W., Hu, Z., Jia, F., Li, Y., Xu, H., . . Qu, Z. (2014). Experiences of stigma and discrimination among caregivers of persons with Schizophrenia in China: A field survey. Plos One, 9(9). doi: 10.1371/journal.pone.0108527 